\title{
BMJ Open Incremental effect of non-invasive oscillating device on chest physiotherapy in critically ill children: a cross-over randomised trial
}

\author{
Atsushi Kawaguchi (D , ${ }^{1,2}$ Gabrielle Bernier, ${ }^{3}$ Andy Adler, ${ }^{4}$ Guillaume Emeriaud, ${ }^{1}$ \\ Philippe A Jouvet ${ }^{1}$
}

To cite: Kawaguchi A, Bernier G, Adler A, et al. Incremental effect of non-invasive oscillating device on chest physiotherapy in critically ill children: a crossover randomised trial. BMJ Open 2020;10:e038648. doi:10.1136/ bmjopen-2020-038648

- Prepublication history and additional material for this paper are available online. To view these files, please visit the journal online (http://dx.doi. org/10.1136/bmjopen-2020038648).

Received 18 March 2020 Revised 22 July 2020 Accepted 25 August 2020

Check for updates

(C) Author(s) (or their employer(s)) 2020. Re-use permitted under CC BY-NC. No commercial re-use. See rights and permissions. Published by BMJ.

${ }^{1}$ Pediatrics, University of Montreal, Montreal, Quebec, Canada

${ }^{2}$ Pediatrics, University of Ottawa, Children's Hospital Eastern Ontario, Ottawa, Ontario, Canada ${ }^{3}$ Faculty of Medicine, University of Montreal, Montreal, Quebec, Canada

${ }^{4}$ Systems and Computer Engineering, Carleton University, Ottawa, Ontario, Canada

Correspondence to Dr Philippe A Jouvet philippe.jouvet@umontreal.ca

\section{ABSTRACT}

Introduction Chest physiotherapy (CPT) and intrathoracic percussion ventilation have been recognised as to encourage dislodging the secretions; nonetheless, the tolerance to the procedure and its efficiency have not been proven to be sufficient.

Method and analyses This study aims to examine the tolerance, feasibility and physiological effects in airway clearance by using a novel extrathoracic non-invasive oscillating transducer device (NIOD) in critically ill children. A two-stage cross-over randomised controlled study in a paediatric intensive care unit in a Canadian Academic Children's Hospital will be applied. Children under 24 months old, for whom CPT is prescribed for airway clearance, will be included. The study consists of two stages; (1) Stage 1 'Frequency Level': we will apply two different frequencies of the NIOD $(40 \mathrm{~Hz}$ vs $60 \mathrm{~Hz})$ for 12 min each, on each patient 3 hours apart, and (2) Stage 2 'NIOD versus CPT': we will implement NIOD and CPT alternatingly for 3 hours apart. The order of the procedures will be randomly allocated for each case. We will compare the average $\Delta$ changes of tidal lung volume measured by a 3D imaging system and regional lung functions using electrical impedance tomography, between the two different frequencies and between the NIOD periods and the CPT periods. We will also examine tolerance by seeing COMFORT Scales and related complications during the procedures. We estimate necessary sample size as 6 for each arm (Total 12 cases) for stage 1 and 48 cases for Stage 2, with power of 0.8 and alpha of 0.05 .

Ethics and dissemination This study has been approved by the Health Research Ethics Board of University of Montreal, Canada (REB number: 2020-2471). We will disseminate our findings through peer-reviewed publications and conference presentations in paediatric or/ and critical care fields.

Trial registration number ClinicalTrials.gov Registry (NCT03821389).

\section{INTRODUCTION}

Anatomical feature of infants and small children involves disproportionally high airway resistance due to their nasal breathing and relatively small radius of the upper airway. Consequently, thick and viscous secretions
Strengths and limitations of this study

- This is the very first randomised controlled study to assess the effect of an extrathoracic oscillating device in critically ill children.

- By applying a stepwise study, we will be able to explore a variety of important clinical and physiological effects.

- The study will also reveal the physiological effect of chest physiotherapy by using several respiratory monitoring devices including $3 \mathrm{D}$ imaging system and electrical impedance tomography.

in respiratory illness such as bronchiolitis or pneumonia reduce further the lumen airway, by increasing the resistance drastically. ${ }^{1}$ It decreases cough efficiency and causes air trapping, leading to severe dyspnoea and atelectasis. The poorly developed compensatory collateral ventilation in infants and small children also leads to easier development of respiratory failure. ${ }^{2}$

Chest physiotherapy (CPT) has been proposed to assist in the clearance of tracheobronchial secretions. The goal of CPT is to clear the mechanical airway obstruction, reduce airway resistance, enhance gas exchange and reduce the work of breathing eventually. Techniques performed in the conventional CPT such as a chest percussion, vibration and chest shaking may help facilitate to mobilise the secretions towards the trachea with a trigger coughing that could help to remove the secretions. However, it is also recognised that the conventional CPT may have drawbacks such as poor tolerance in infants with increasing agitation during the procedure, due to which we may not be able to provide effective and consistent techniques.

Although CPT still has been playing an important role in an intensive care as an 
alternative practice of airway management, a recent systematic review particularly looking at the hospitalised children with respiratory distress or failure reported that there is no sufficient evidence for us to provide CPT in a routine practice. ${ }^{3-7}$ At the same time, we need to be aware of the significant heterogeneity of the results among the evidence and inconsistency of the procedure techniques used. $^{8}$

Extrathoracic non-invasive oscillating devices (NIODs) are designed to interrupt the expiratory airflow, vibrating at variable frequencies and intensities as set by the operator to ensure the individual's comfort and associated concordance. A combination of strong and weak coupling to the curved chest wall can transmit vibrations to both deep and shallow tissues. Peristaltic action due to longitudinal waves could provide a better clearance. Generally, NIOD requires less patient cooperation and could be used without interruptions such as due to coughing or suctioning of secretion. Frequencer (Dymedso, Montreal, Canada) is a NIOD distinguishing itself by being able to locally target the affected area, instead of influencing entirely the patients' lungs. ${ }^{910}$ The safety has been attested particularly in patients with cystic fibrosis. Although no published data being available, it has also explored in infants in which good tolerance and no apparent adverse events were reported (via personal communication, Dr Freddy Schwab, Germany). However, to date, no well-designed controlled study has been performed for critically ill or injured infants and small children with respiratory distress.

This stepwise cross-over randomised controlled study aims to examine the feasibility, patients' tolerance on the NIOD (Frequencer) and the physiological effects including air distribution of the lung in critically ill children requiring airway clearance.

\section{METHODS}

\section{Study setting}

A paediatric intensive care unit (PICU) in a Canadian Academic Children's Hospital.

\section{Eligibility criteria}

Screening of eligible patients and informed consent procedure

We will identify potential subjects in the PICU every morning during the study period. We will obtain the informed consent (IC) from parents or guardians as soon as possible from the identification of the patients. The research assistant will obtain the IC using a pre-specified form.

\section{Inclusion criteria}

All the patients under 2years old admitted to the PICU during the study period will be screened. We will not set any restriction regarding the timing of prescription of CPT (ie, length of PICU stay before screening) for the screening. We will include only if CPT is expected to be used as a management at least for the next 24 hours in the
PICU from the time of inclusion. For instance, if CPT will be expected to be discontinued from the management in a day, we will exclude them from the inclusion. CPT can be prescribed for airway clearance with any aetiology such as atelectasis at the directions of bedside paediatric intensivists in charge on the study date. We will only include patients whose oxygenation is stable $\left(\mathrm{SpO}_{2}>90 \%\right)$ with less than 0.60 of fractional inspired oxygen $\left(\mathrm{FiO}_{2}\right)$.

\section{Exclusion criteria}

We will exclude the cases if they meet at least one exclusion criteria below.

- CPT order is expected to be discontinued within 24 hours from the inclusion timing.

- CPT is not ordered for the airway clearance purpose.

- $\mathrm{SpO}_{2}$ is not stable $\left(\mathrm{SpO}_{2} \leq 90 \%\right)$ with $\mathrm{FiO}_{2} \geq 0.60$ for the ventilated patients including patients on noninvasive ventilation (NIV), at least for previous 1 hour from the screening.

- $\mathrm{SpO}_{2}$ is not stable $\left(\mathrm{SpO}_{2} \leq 90 \%\right)$ with $\mathrm{FiO}_{2} \geq 0.60$ for the patients on high-flow nasal cannula (HFNC), at least for previous 1 hour from the screening.

- Bradycardia (heart rate $<80$ beats per minute) at any interventions at least for 24 hours prior to the screening.

- Patients with known pneumothorax, osteomyelitis in the PICU admission.

- A known pulmonary hypertension with treatment(s) underway.

- Thoracotomy within 1 month before the screening.

- A known recent/unhealed rib fractures.

- Known skin injury of chest wall.

- No obtain of IC.

- Brain death or vegetated states.

- Patient was already included in the study.

\section{Interventions}

Each subject will receive the NIOD and the standardised CPT for this study. NIOD will be implemented on four different parts of the chest walls, 3 min for each part and $12 \mathrm{~min}$ in total per each session. Left and right front and posterior chest walls will be stimulated, particularly, on the anterior chest, intercostal spaces 1-2 above nipple line and lateral side of the mid-clavicular line 1-2 below intercostal spaces. The intensity of the NIOD can be selected between $80 \%$ and $100 \%$, which is prespecified on the machine. There are three selections of the membrane available. We will use the largest membrane for all the cases. The procedure using the NIOD will be standardised. We will provide proper training to the caregivers using a procedure protocol of the NIOD. The detailed mechanism of the NIOD can be found in the online supplemental document 1 .

\section{Criteria for discontinuing allocated interventions}

For this study, the Data and Safety Monitoring Board (DSMB) will be in place to independently supervise any potential side effects. It will include two clinicians 
expertised in paediatric pulmonology in the study institution. The DSMB will follow the study completion and review any significant potential complications related to the intervention with NIOD. Any occurrence of pneumothorax, need for unplanned intubation, life-threatening event, during or in the 2 hours following the study, will be declared to and reviewed by the DSMB, which will independently decide on the need to stop or continue the study.

\section{Relevant concomitant care}

\section{Suctioning and other interventions}

As per the PICU protocol, subjects will have endotracheal suctioning as needed. Any other potential interventions such as nebulisation of hypertonic saline and bronchodilator and that with a metered-dose inhaler can be given without any restrictions.

\section{Positioning}

All the procedure will be applied with any body positioning such as sitting and prone positions. Caregivers can also change the positioning as needed during the procedures.

Vital signs, ventilator parameters, end tidal $\mathrm{PCO}_{2}$ $\left(\mathrm{EtCO}_{2}\right)$ and electrical impedance tomography (EIT) will be measured with supine position.

\section{Chest physiotherapy}

CPT will be defined as an assistant strike to the chest wall repeatedly with a cupped hand in specific places. The standard CPT, particularly in the stage 2 below, will be performed by a physiotherapist as per prespecified protocol for 15-20 min. Our standard CPT applies chest percussion with a clapping of the chest and positioning to facilitate the drainage.

\section{Outcomes}

Stage 1

\section{Primary outcome}

Mean changes in respiratory tidal volume (right before and after the procedure), which will be measured either by the ventilator in mechanically invasively ventilated children or by a 3D imaging system in spontaneous breathing children with/without non-invasive mechanical ventilator such as HFNC. ${ }^{11}$ In brief, an algorithm will calculate the tidal volume by measuring the thorax-abdomen difference in volume between end of expiration and inspiration, from a camera that acquires RGB-D infant's videos (colour plus depth). Moreover, video recordings are synchronised with the hospital timeserver, therefore synchronised with all other acquisitions such as vital signs, measurements in ventilator and blood gas performed at the PICU.

\section{Secondary outcomes}

We will also record the vital sign values including blood pressures, heart rates, oxygen saturations, respiratory rate, temperature and neurological status, including patient comfort level, oxygen saturation and work of breathing. Estimated regional lung volume and other parameters measured by $\mathrm{EIT}^{12-14}$ and the values of $\mathrm{EtCO}_{2}$ and its waveform will also be collected.

\section{Stage 2}

We will compare the two groups (CPT only and CPT with NIOD) for the following outcome items.

\section{Primary outcome}

Mean modified COMFORT Scale 2 min from the initiation of the procedure, ${ }^{15} 16$ which is a behavioural, unobtrusive method of measuring distress in unconscious and ventilated infants, children and adolescents using eight different indicators.

\section{Secondary outcomes}

Mean of respiratory tidal volume. Vital sign values including blood pressures, heart rates, oxygen saturations, respiratory rate, temperature and neurological status, including patient comfort level, caregiver estimation of the work of breathing, estimated lung volume and other parameters measured by EIT and the values of $\mathrm{EtCO}_{2}$ and its waveform, and clinical respiratory severity score. $^{27}$

\section{Sample size and feasibility of enrolment}

For the stage 1, since we do not have pre-existing data regarding the effects on different frequencies in small children with the new devices, we will use expiratory tidal ventilatory volume as a primary outcome and apply it for the sample size calculation. The estimated improvement of the tidal volume is $0.8 \mathrm{~mL} / \mathrm{kg}$ in the group with $40 \mathrm{~Hz}$ and is $0.4 \mathrm{~mL} / \mathrm{kg}$ in the group with $60 \mathrm{~Hz}$. We set up the expected within-group variances of $0.2 \mathrm{~mL} / \mathrm{kg}$, which gives 12 total sample size. For stage 2, we estimate the necessary sample size as 24 for each arm (total 48 cases), with a potential effect difference of 2.5 points of COMFORT Scale, power of 0.8 , alpha of 0.05 and SD of $3.0(15,16)$. We will include 60 patients (30 in each arm) to take into account $20 \%$ attrition (ie, any technical or patient reason that may result in the default in data analyses). Children under 2 years old represent $66 \%$ of our PICU admissions (ie, $1000 * 0.66=660 /$ year) and at least $50 \%$ of them have CPT during the PICU stay, which gives us 330 children/ year. Considering that all the patients admitted to the PICU during the study period will be screened and our consent rate has been around $80 \%$, it can be feasible to recruit the 60 patients within at least a year.

\section{Allocation}

Sequence generation and allocation concealment Stage 1

Patients satisfying the eligibility criteria will have their baseline data collected before randomisation. Different frequencies of NIOD will be applied each time every 3 hours. Patients will be randomised into one of the two arms: $40 \mathrm{~Hz}$ versus $60 \mathrm{~Hz}$ (figure 1). In the adult study, $20-60 \mathrm{~Hz}$ is most widely used (unpublished data from Dymedso). ${ }^{9}{ }^{10}$ We will apply $40 \mathrm{~Hz}$ and $60 \mathrm{~Hz}$ considering 
RANDOMISATION
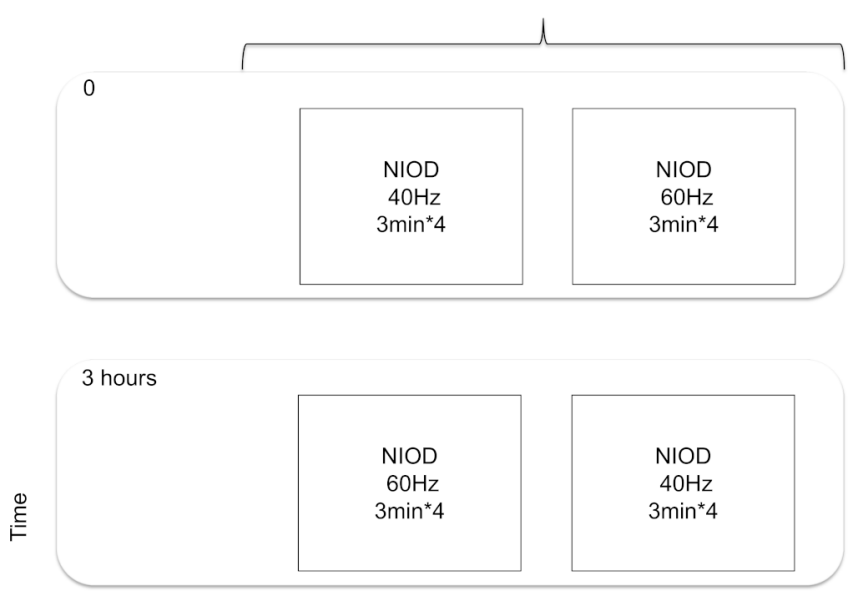

Figure 1 Schematic overview for the stage 1. NIOD, noninvasive oscillating transducer device.

that the thorax of infants and small children is more compliant than the adult, therefore, we could expect that a higher frequency should be applied in children. Randomisation will be carried out by the independent research assistant in CHU Sainte Justine (CHUSJ), using a computerised procedure. Random allocation will be generated by an independent investigator in an equal number assigned to each intervention. No stratification will be applied.

\section{Stage 2}

The frequency which will be selected (ie, more welltolerated with/without most effective) in the stage 1 will be applied (figure 2). If there are no significant differences among the two frequencies in the stage 1, we will apply $40 \mathrm{~Hz}$ for the stage 2 based on the previous evidences in adult and older children using the already commercialised device. The time of the intervention implemented will be determined based on the time of

At least 3 hours from the previous CPT

\section{RANDOMISATION}

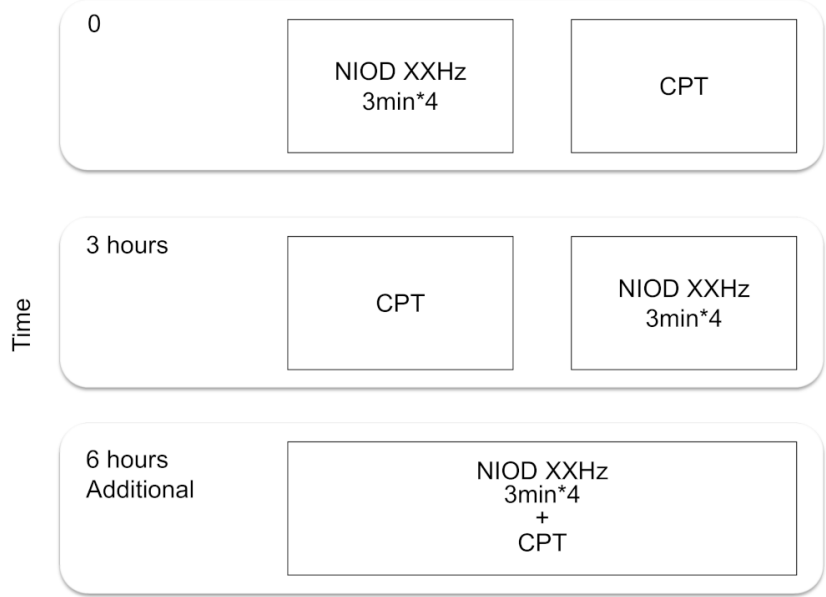

Figure 2 Schematic overview for the stage 2. CPT, chest physiotherapy; NIOD, non-invasive oscillating transducer device. the CPT scheduled by a physiotherapist. Patients will be randomised into one of the two arms. Randomisation will be carried out by the independent research assistant in CHUSJ, using a computerised procedure. Simple randomisation will be applied. Random allocation will be generated by an independent investigator.

\section{Data collection, management and analyses \\ Timing of the data measurement}

The data measured by the ventilator, EIT and 3D video recording system will be collected (1) right before the intervention being initiated, (2) right after the end of the intervention and (3) 30 min after each intervention finished. Vital sign values including $\mathrm{EtCO}_{2}$ waveforms from the beginning of the intervention until $30 \mathrm{~min}$ after the intervention will be extracted from the highresolution database (ie, every minute).$^{18}$

\section{Data elements to be collected}

Chronologically number will be applied for each study subject. Patients' demographics including gender, age, admission date and time, and diagnoses based on 10th revision of the International Classification of Diseases and Related Health Problems codes including chronic and acute diseases. The following items will be recorded (1) before, (2) after each procedure and (3) $30 \mathrm{~min}$ from the procedure: vital signs including blood pressures, heart rates, oxygen saturations, respiratory rate, temperature, neurological status (eg, Glasgow Coma score, modified COMFORT Scale) and work of breathing (mild, moderate and severe). Signs of increasing work of breathing will be evaluated by two independent investigators (ie, one physician and one respiratory therapist (RT)) based on retraction (intercostal, suprasternal, costal margin, paradoxical abdominal breathing) and accessory muscle use (nasal flaring, sternomastoid contraction and forward posture); expiratory tidal volume, measured positive end-expiratory pressure and peak inspiratory pressure when patients are on invasive mechanical ventilation (InMV) with an endotracheal tube; estimated quadrant regional lung volumes (front right, posterior right, front left and posterior left volumes) and other parameters measured by EIT; EtCO and its waveform; and respiratory severity score. ${ }^{17}$ We will apply our 3D non-invasive imaging system to capture the ventilatory tidal volume. This will be applied not only for the non-intubated patients with NIV or HFNC but also for the patients with InMV. The details of the system and the methods are described in the reference manuscript. ${ }^{11}$ We will quantify the variability of $\mathrm{EtCO}_{2}$ waveform. We could hypothesise that the better the oscillationed air conducts across the chest wall and lung parenchyma, the larger the baseline $\mathrm{EtCO}_{2}$ variability can be observed from the baseline waveform. We will measure and quantify the average variability $(\mathrm{mm} \mathrm{Hg})$ of $\mathrm{EtCO}_{2}$ during each study period. ${ }^{19}$

Since there is no single scale which is well validated for the assessment of 'tolerance' to the invasive procedure in children, we will apply multiple existing scales such as modified COMFORT Scale as a measurement in this 
study. The score will be recorded before, 2 min from the initiation of the procedure, right after the procedure, and $30 \mathrm{~min}$ from the procedure. It was originally designed to assess distress/comfort in ventilated children and has been validated for measuring distress in children admitted to PICU. ${ }^{15} 16$

\section{Patient and public involvement}

No patient or public involvement.

\section{Statistical methods}

For the stage 1, we will describe the background characteristics of the patients, then explore the average outcome values and compare among the two frequency levels. For the stage 2, we will first describe the demographic characteristics of the patients in the two groups. Then, we will compare the outcome values between the CPT and NIOD at 3 hours interval. The additional intervention at 6 hours (ie, CPT+NIOD, figure 2) is not mandatory (will depend on clinical constraints) and will not be considered as part of the main study. A two-sided $p$ value of less than 0.05 will be considered statistically significant. All statistical analyses will be conducted with Stata V.13 (Stata Corp, Texas, USA) or other statistical software(s).

\section{Ethics and dissemination}

Although it has not been well-tested in infants in welldesigned controlled studies, the mechanism of the pressure delivery and the intensity of the delivering pressure/ oscillation should be similar as compared with the existing model for adult patients. We believe there should be no excessive harm as compared with the current standard treatment. To minimise the potential disadvantages of the participants, all the patients will receive standard CPT during the study period. This study has been approved by the Health Research Ethics Board of University of Montreal, Canada (REB number: 2020-2471).

Knowledge translation will be integrated throughout the study. We will engage knowledge users involved in each stage of the project. This will include paediatric intensivists, RTs and physiotherapists. We emphasise our strategy of knowledge application in our existing research platform with relevant professionals, which could allow us to evaluate the outcomes and monitor knowledge use during and after the project. The goal of this research is to increase knowledge and change practice promptly in healthcare professionals. At the end of the project, we will disseminate our findings through peer-reviewed publications and conference presentations in paediatric critical care fields.

Contributors PAJ will coordinate the project team and the implementation of the project's findings into the clinical community. AK and GE will provide the team with advice on methodologies and lead in the dissemination and application of knowledge. GB will contribute by bridging the scientific team and the bedside caregiver. AA will engage the project by providing knowledge and resources of EIT. The data collection will be performed by AK and research assistants. Data analyses will be conducted by AK.

Funding This study has been supported by Dymedso Inc. for the devices and remunerations for the research assistants for data collections. Data and analysis are owned by the investigators. Dymedso Inc. will be informed of the results after the analyses are performed.

Competing interests None declared.

Patient and public involvement Patients and/or the public were not involved in the design, or conduct, or reporting, or dissemination plans of this research.

Patient consent for publication Parental/guardian consent obtained.

Provenance and peer review Not commissioned; externally peer reviewed.

Open access This is an open access article distributed in accordance with the Creative Commons Attribution Non Commercial (CC BY-NC 4.0) license, which permits others to distribute, remix, adapt, build upon this work non-commercially, and license their derivative works on different terms, provided the original work is properly cited, appropriate credit is given, any changes made indicated, and the use is non-commercial. See: http://creativecommons.org/licenses/by-nc/4.0/.

ORCID iD

Atsushi Kawaguchi http://orcid.org/0000-0002-2209-5835

\section{REFERENCES}

1 Ralston SL, Lieberthal AS, Meissner HC, et al. Clinical practice guideline: the diagnosis, management, and prevention of bronchiolitis. Pediatrics 2014;134:e1474-502.

2 Nelson textbook of pediatrics 20th edition; respiratory pathophysiology and regulation 2016:1981-93.

3 Hawkins $\mathrm{E}$, Jones $\mathrm{A}$. What is the role of the physiotherapist in paediatric intensive care units? A systematic review of the evidence for respiratory and rehabilitation interventions for mechanically ventilated patients. Physiotherapy 2015;101:303-9.

4 Roqué i Figuls M, Giné-Garriga M, Granados Rugeles C, et al. Chest physiotherapy for acute bronchiolitis in paediatric patients between 0 and 24 months old. Cochrane Database Syst Rev 2016;2:CD004873.

5 Chaves GS, Freitas DA, Santino TA, et al. Chest physiotherapy for pneumonia in children. Cochrane Database Syst Rev 2019;1:CD010277.

6 Hough JL, Flenady V, Johnston L, et al. Chest physiotherapy for reducing respiratory morbidity in infants requiring ventilatory support. Cochrane Database Syst Rev 2008;3:CD006445.

7 Lauwers E, Ides K, Van Hoorenbeeck K, et al. The effect of intrapulmonary percussive ventilation in pediatric patients: a systematic review. Pediatr Pulmonol 2018;53:1463-74.

8 Morrison L, Milroy S. Oscillating devices for airway clearance in people with cystic fibrosis. Paediatr Respir Rev 2018;25:30-2.

9 ReG DS, Galli F, Rigamonti MG, et al. Influence of operating frequency and amplitude in the Frequencer ${ }^{\mathrm{TM}}$ to treat cystic fibrosis.

10 Cantin AM, Bacon M, Berthiaume Y. Mechanical airway clearance using the frequencer electro-acoustical transducer in cystic fibrosis. Clin Invest Med 2006;29:159-65.

11 Rehouma H, Noumeir R, Bouachir W, et al. 3D imaging system for respiratory monitoring in pediatric intensive care environment. Comput Med Imaging Graph 2018;70:17-28.

12 Dmytrowich J, Holt T, Schmid K, et al. Mechanical ventilation guided by electrical impedance tomography in pediatric acute respiratory distress syndrome. J Clin Monit Comput 2018;32:503-7.

13 Lupton-Smith A, Argent A, Rimensberger P, et al. Prone positioning improves ventilation homogeneity in children with acute respiratory distress syndrome. Pediatr Crit Care Med 2017;18:e229-34.

14 Vogt B, Löhr S, Zhao Z, et al. Regional lung function testing in children using electrical impedance tomography. Pediatr Pulmonol 2018;53:293-301.

15 van Dijk M, Peters JWB, van Deventer P, et al. The comfort behavior scale: a tool for assessing pain and sedation in infants. Am J Nurs 2005;105:33-6.

16 Ista E, van Dijk M, Tibboel D, et al. Assessment of sedation levels in pediatric intensive care patients can be improved by using the COMFORT "behavior" scale. Pediatr Crit Care Med 2005;6:58-63.

17 Cambonie G, Milési C, Fournier-Favre S, et al. Clinical effects of heliox administration for acute bronchiolitis in young infants. Chest 2006;129:676-82.

18 Brossier D, El Taani R, Sauthier M, et al. Creating a high-frequency electronic database in the PICU: the perpetual patient. Pediatr Crit Care Med 2018;19:e189-98.

19 Ruiz de Gauna S, Leturiondo M, Gutiérrez JJ, et al. Enhancement of capnogram waveform in the presence of chest compression artefact during cardiopulmonary resuscitation. Resuscitation 2018;133:53-8. 